\title{
Lymphangiography: Forgotten Tool or Rising Star in the Diagnosis and Therapy of Postoperative Lymphatic Vessel Leakage
}

\author{
Sebastian Kos · Harald Haueisen • Ulrich Lachmund • \\ Thomas Roeren
}

Published online: 17 May 2007

(C) Springer Science+Business Media, LLC 2007

\begin{abstract}
Since the advent of computed tomography, numbers and expertise in Lymphangiography (LAG) have markedly dropped. The intention of our study was to demonstrate the persisting diagnostic and therapeutic impact of LAG on the postoperative patient with known or suspected lymphatic vessel leakage. Between May 1, 1999, and April 30, 2006, we investigated pedal lipiodol-LAGs (18 monopedal, 2 bipedal) on 22 patients (16 male, 6 female) with known or suspected postoperative chylothorax, chylaskos, lymphocele, or lymphatic fistula. Ages varied from 26 to 81 years. The spectrum of operative procedures was broad: 6 thoracic, 5 abdominal, and 11 peripheral operations were performed. In 20 patients who underwent mono- or bipedal LAG for lymphatic vessel injury, we were able to demonstrate the specific site of leakage in 15 cases $(75 \%)$ and found signs of extravasation in 5 patients (25\%). Furthermore, in 11 patients $(55 \%)$ we were able to avoid surgery because of closure of the leak after LAG. As the conservative therapeutic approach usually takes $2-3$ weeks to reveal its therapeutic effects, $73.3 \%$ (11/15) of the patients who were not reoperated before this hallmark was passed did not need any further operation. Our study clearly demonstrates that even in the decades of modern cross-sectional imaging, classic LAG is a powerful and
\end{abstract}

S. Kos $\cdot$ H. Haueisen $\cdot$ T. Roeren

Institute of Radiology, Kantonsspital Aarau, 5001 Aarau,

Switzerland

U. Lachmund

Institute of Radiology, Universitätsspital Zürich, Rämistrasse 100, 8091 Zürich, Switzerland

S. Kos $(\bowtie)$

Universitätsspital Basel, Petersgraben 4, 4031 Basel, Switzerland

e-mail: skos@gmx.de highly reliable tool to visualize and even assist occlusion of the postoperatively damaged lymphatic vessel and may thereby avoid the need for reoperation.

Keywords Lymphangiography · Postoperative ·

Chylaskos $\cdot$ Lymphocele $\cdot$ Chylothorax $\cdot$ Lymphatic fistula

\section{Introduction}

Classic Lymphangiography (LAG), used for decades, has the capability to detect internal architectural derangements within normal-sized lymph nodes and, in addition, can demonstrate and prove the lymphatic origin of a detected fluid collection as seen in chylaskos, lymphocele, chylothorax, or lymphatic fistula in the postoperative patient [1]. Using lipiodol as the contrast agent, its potential to induce granulomatous reactions when extravasating has been demonstrated [2] when assisted by additive procedures like compression, drainage, and total parenteral nutrition [3-6]. The intention of our study was to demonstrate the diagnostic and therapeutic impact of LAG on the postoperative patient with known or suspected lymphatic vessel leakage.

\section{Materials and Methods}

Between May 1, 1999, and April 30, 2006, we investigated pedal lipiodol-LAGs (18 monopedal, 2 bipedal) on 22 patients (16 male, 6 female) with known or suspected postoperative chylothorax, chylaskos, lymphocele, or lymphatic fistula. Ages varied from 26 to 81 years. The spectrum of operative procedures performed was broad: 6 thoracic, 5 abdominal, and 11 peripheral operations (Table 1). 
Table 1 Indication and spectrum of operative procedures performed prior to LAG, resulting postoperative leakage type, and diagnostic/ therapeutic impact of LAG (diag./ther.)

\begin{tabular}{|c|c|c|c|c|c|}
\hline Operative procedure & Underlying disease/indication & $\begin{array}{l}\text { No. } \\
\text { cases }\end{array}$ & Leakage type & Diag./ther. & Duration \\
\hline \multirow[t]{2}{*}{ Pneumectomy } & \multirow[t]{2}{*}{ Bronchial carcinoma } & \multirow[t]{2}{*}{2} & \multirow[t]{2}{*}{ Chylothorax } & $+/+$ & 11 days \\
\hline & & & & $\mathrm{TF}$ & 15 days \\
\hline \multirow[t]{2}{*}{ Nephrectomy } & Renal cell carcinoma & 1 & Chylaskos & $++/+$ & 5 days \\
\hline & Polycystic kidney disease & 1 & Chylothorax & $(+)+/-$ & $5 \mathrm{wk}$ \\
\hline \multirow[t]{3}{*}{ Esophagectomy } & \multirow[t]{2}{*}{ Esophageal carcinoma } & \multirow[t]{2}{*}{2} & \multirow[t]{2}{*}{ Chylothorax } & $++/ 14$ days & 14 days \\
\hline & & & & $++/ 7$ days & 18 days \\
\hline & Melanoma & 1 & Chylothorax & $++/ 21$ days & 13 days \\
\hline \multirow[t]{6}{*}{ Lymphadenectomy } & \multirow[t]{3}{*}{ Melanoma } & \multirow[t]{3}{*}{3} & \multirow[t]{3}{*}{ Lymph. fistula } & $++/+$ & 17 days \\
\hline & & & & $++/+$ & 12 days \\
\hline & & & & $\mathrm{TF}$ & 5 days \\
\hline & M. Hodgkin & 1 & Lymph. fistula & $+/+$ & $8 \mathrm{wk}$ \\
\hline & Vulval carcinoma & 1 & Lymph. fistula & $(\mathrm{TF})++/-$ & 17 days \\
\hline & Penile carcinoma & 1 & Lymph. fistula & $++/+$ & 6 days \\
\hline Thymectomy & Myasthenia gravis & 1 & Chylothorax & $++/+$ & 6 days \\
\hline \multirow[t]{2}{*}{ Endoaneurysmoraphy } & \multirow[t]{2}{*}{ Aneurysmal disease } & 1 & Lymph. fistula & $++/+$ & 22 days \\
\hline & & 1 & Lymphocele & $++/-$ & $16 \mathrm{wk}$ \\
\hline $\begin{array}{l}\text { Semicastration + retroperitoneal } \\
\text { lymphadenectomy }\end{array}$ & Embryonic carcinoma & 1 & $\begin{array}{l}\text { Chylothorax+ } \\
\text { Chylaskos }\end{array}$ & $\begin{array}{l}++/+ \text {, re-exam. } 8 \\
\quad \text { wk }\end{array}$ & 16 days \\
\hline Prostatectomy & Prostate carcinoma & 1 & Lymphocele & $++/ 14$ days & 3 wk \\
\hline \multicolumn{6}{|l|}{ Bypass } \\
\hline $\mathrm{ACVB}$ & Coronary heart disease & 1 & Lymph. fistula & $++/+$ & $22 \mathrm{wk}$ \\
\hline Fem-POP & $\begin{array}{l}\text { Peripheral artery occlusive } \\
\text { disease }\end{array}$ & 1 & Lymph. fistula & +/4 days, for. body & $6 \mathrm{wk}$ \\
\hline Partial gastrectomy & Gastric carcinoma & 1 & Chylothorax & $+/+$ & 13 days \\
\hline Soft tissue resection & Thigh seroma & 1 & Lymphocele & $++/-$ & $6 \mathrm{wk}$ \\
\hline
\end{tabular}

Note. + , indirect visualization; ++ , direct visualization of the leakage site; days, reoperation at 4-21 days after LAG; lymph., lymphatic; TF, technical failure; ( ), bipedal LAG; for. body, foreign body; re-exam. 8 wk, re-examination after 8 weeks; ACVB = CAVB; Duration of lymphatic leakage before LAG was performed: 5 days-22 weeks

The lymphatic vessel leakage had persisted for between 5 days and 22 weeks, when LAG was performed (Table 1). In those cases with thoracic or abdominal drainage in place, the daily amount of drainage ranged between 200 $\mathrm{ml} / \mathrm{d}$ and $3 \mathrm{~L} /$ day. Therefore we monitored the sensitivity of our LAGs to directly visualize the site of leakage and the presence of abnormal lymphatic structures such as lymphoceles and fistulas or indirectly reveal a pathologic stop of lipiodol distribution and contrast runoff into extracorporeal drainage reservoirs. In addition, we monitored the outcome of conservative treatment of those conditions with LAG, assisted by additive procedures such as compression, drainage, and total parenteral nutrition.

All LAGs were performed with the written informed consent of the patient and in the absence of contraindications. The patient is brought into a supine position with bare feet. After local cutaneous disinfection (Braunoderm; Braun), we inject a mixture of $2 \mathrm{ml}$ of $1 \%$ Lidocaine and 2 $\mathrm{ml}$ of methylene blue dye (1\% methylthionium chloride, Methylenblau Vitis; Neopharma $\mathrm{GmbH}$ ) into the cutaneous and subcutaneous tissues of the first and second interdigital space. After $10 \mathrm{~min}$ of exercising the foot the course of the lymphatic vessels is identified through the skin. Via a longitudinal cutaneous incision a vessel lateral to the base of the first metatarsal is exposed, but also a more proximal vessel near the ankle may be chosen. The surrounding tissues are stripped, thereby giving good access to the lymphatic vessel, which is then cannulated (De Roo needles Nos. 35 and 40 with spring and mandarin). Needle and infusion line are secured with adhesive stripes (Steri-Strip; $3 \mathrm{M}$ Health Care). Once having secured access to the vessel, we apply up to $1 \mathrm{ml} / 10 \mathrm{~kg}$ body weight per foot, not exceeding a total volume of $14 \mathrm{ml}$ of lipiodol $(48 \%$ iodinated glycerol ester), using an injector with an injection speed of 4-7 ml/h per side. After the injection is completed the materials are removed and the wound is cleansed, su- 


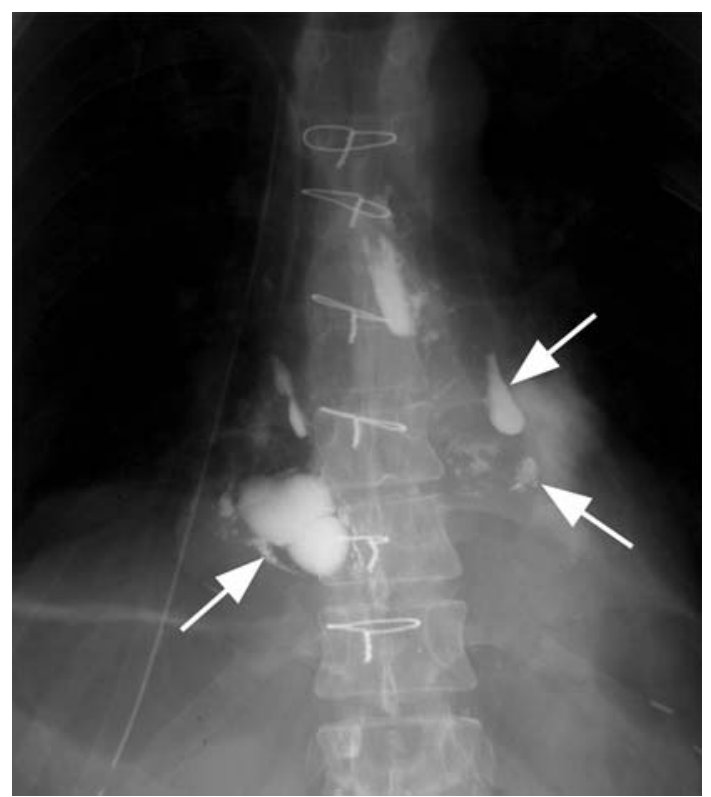

Fig. 1 Chylothorax after esophagectomy in the case of esophageal carcinoma. Leakage of the thoracic duct at the level Th6/Th7. Note the extravasated lipiodol (arrows)
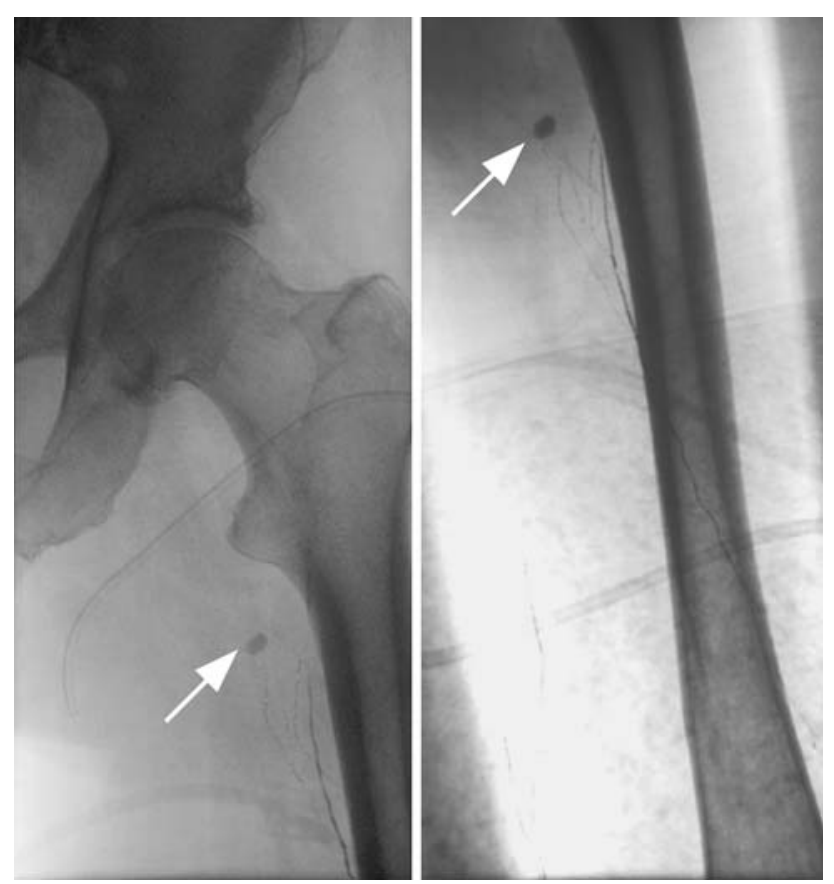

Fig. 2 Lymphatic fistula of the left thigh after resection of a melanoma of the first toe with additive inguinal and suprainguinal lymphadenectomy. Note the extravasated lipiodol (arrows)

tured, and covered with adhesive bands. Sutures may be removed at postinterventional days $7-10$. The application is followed by complete fluoroscopic documentation of the contrast runoff toward the venous angle (filling phase) plus late-phase imaging after about $24 \mathrm{~h}$ (nodal phase). In cases
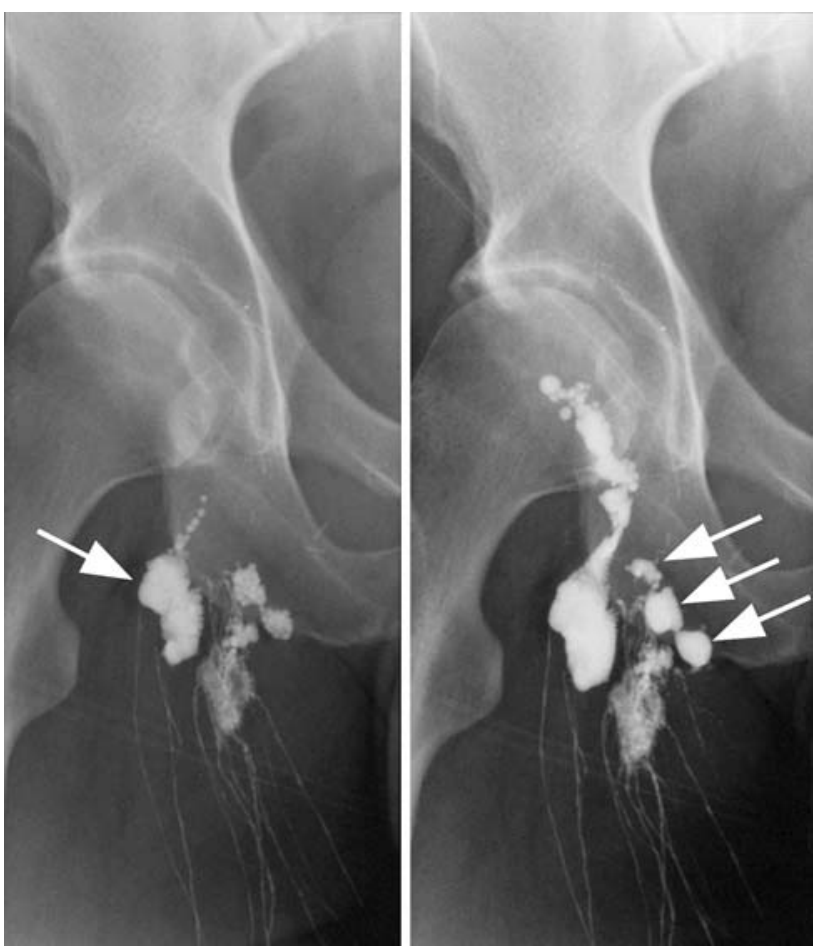

Fig. 3 Lymphatic fistula after resection of a melanoma of the right thigh with additive inguinal and suprainguinal lymphadenectomy. Note the lipiodol leakage (arrows)

of cutaneous lymphatic fistulas the known cutaneous porus is identified using an $\mathrm{x}$-ray absorbent marker prior to documentation.

\section{Results}

Of the 22 patients we intended to diagnose and treat we initially failed to cannulate $2(9.1 \%)$ for contrast-medium application, one due to lymphedema and one due to very thin and fragile lymphatic vessels. Twenty patients (90.9\%) underwent lipiodol-LAG (18 monopedal, 2 bipedal). Of the 20 patients who were lymphangiographied, 7 presented with chylothorax, 8 with a lymphatic fistula, 3 with a lymphocele, and 1 with chylaskos. In one case chylothorax plus chylaskos was known.

LAG in 15 of these $20(75.0 \%)$ patients directly determined the sites of femoral, thoracic, and abdominal contrast leakage, fistula, or lymphocele, respectively (Figs. 1$3)$.

In five patients (25.0\%) there were only indirect signs of lymphatic vessel injury such as lipiodol detection in the drainage reservoir (one case), pathologic disruption of the centripetal contrast medium distribution with consecutive masking of the extravasating lipiodol by dilution (two cases), and atypical lymphatic vessels as a sign of lym- 
Table 2 Diagnostic and therapeutic results of LAG in the types of lymphatic vessel leakage investigated

\begin{tabular}{|c|c|c|c|c|c|}
\hline & Chylothorax & Chylaskos & Chylothorax + chylaskos & Lymphatic fistula & Lymphocele \\
\hline No. cases & 7 & 1 & 1 & 8 & 3 \\
\hline Direct visualization & $4 / 7(57.1 \%)$ & $1 / 1(100 \%)$ & $1 / 1(100 \%)$ & $6 / 8(75 \%)$ & $3 / 3(100 \%)$ \\
\hline Indirect visualization & $3 / 7(42.9 \%)$ & - & - & $2 / 8(25 \%)$ & - \\
\hline Therapeutic success & $3 / 7(42.9 \%)$ & $1 / 1(100 \%)$ & $1 / 1(100 \%)$ & $6 / 8(75 \%)$ & $0 / 3(0 \%)$ \\
\hline Reoperation $<4$ wk after LAG & $3 / 7(42.9 \%)$ & - & - & $1 / 8(12.5 \%)$ & $1 / 3(33.3 \%)$ \\
\hline Success without early reoperation & $3 / 4(75 \%)$ & - & - & $6 / 7(85.7 \%)$ & $0 / 2(0 \%)$ \\
\hline Therapeutic failure & $1 / 7(14.3 \%)$ & - & - & $1 / 8(12.5 \%)$ & $3 / 3(100 \%)$ \\
\hline
\end{tabular}

phatic collateralization (two cases). Of the 20 patients who were lymphangiographied, $11(55.0 \%)$ needed no surgical reintervention and the lymphatic leakage was stopped by conservative treatment (e.g. additional compression, drainage, and total parenteral nutrition) alone. Thoracocenteses, if used in the conservative approach, were removed when drainage was $<100 \mathrm{ml} /$ day. Five of those 20 patients were operated before $(4,7,14,14$, and 21 days post-LAG) the completion of 3 weeks of conservative treatment.

In the prostatectomy patient, the infected lymphocele was surgically fenestrated and a Salem drainage was inserted 14 days after LAG. In the patient with the Fem-Pop bypass, the foreign body (catheter fragment) that had been documented by x-ray during LAG was removed and the wound was debrided 4 days after LAG.

In the patient who underwent esophagectomy due to melanoma, rethoracotomy and surgical occlusion of the thoracic duct were performed at day 21 after LAG. In the two patients who underwent esophagectomy for carcinoma of the esophagus, rethoracotomy and surgical occlusion of the thoracic duct followed at days 7 and 14 after LAG.

Eleven of $15(73.3 \%)$ cases in which LAG and a further conservative treatment regimen were not interrupted by early surgery were cured with conservative treatment alone.

In the subgroup analysis chylothorax (75\%), chylaskos $(100 \%)$, chylothorax plus chylaskos (100\%), and lymphatic fistula $(85.7 \%)$ showed very high rates of therapeutic success without the need for further surgical intervention (Table 2). In contrast, none of the three lymphocele cases was treated sufficiently with conservative management alone. Chylaskos, chylothorax plus chylaskos, and lymphocele were directly visualized in $100 \%$ of cases, compared with $57.1 \%$ of the chylothoraces and $75 \%$ of the lymphatic fistulas.

In one case the remaining part of a drainage catheter was detected as a foreign body in the soft tissue of the thigh by fluoroscopy, which led to early reoperation (day 4 after LAG) with extraction of the foreign body and sealing of the lymphatic fistula.
In the case with combined chylothorax and chylaskos after semicastration and retroperitoneal lymphadenectomy due to embryonic carcinoma, the initial LAG led to a therapeutic success but recurrence of symptoms made a diagnostically and therapeutically successful LAG necessary after 8 weeks.

\section{Discussion}

The use of pedal LAG to demonstrate lymphatic vessel leakage is well known. Sachs et al. clarified in 1991 that in the case of LAG in patients with suspected laceration of the thoracic duct, computed tomography is of little additional value [7]. The spectrum of known sites of postoperative lymphatic vessel leakage is broad, as seen in our study, and may therefore lead to different manifestations such as chylous ascites (a.k.a. chylaskos), chylothorax or even chyluria, lymphocele, and lymphatic fistula [8-16]. It must be emphasized that these manifestations may occur not only postoperatively but also in the course of disease as a complication or even congenitally [17, 18]. As of today there are two major approaches to the therapy of identified postoperative lymphatic vessel leakage: operative and nonoperative treatment, with, e.g., the operative treatment of chylothorax through thoracotomy having a high mortality, up to $25 \%$ [19, 20].

Video-assisted thoracic surgery (VATS) may also be used for clipping or ligation of the thoracic duct, as it is safer and less invasive than open thoracotomy. However, this procedure is not widely used, as a switch to the open technique may be necessary if leakage visualization fails endoscopically [21].

Spencer and Maloney initially recommended conservative treatment of chylothorax for at least 3-4 weeks before considering surgery [22]. This was specified by Selle et al., who emphasized that cases with a daily drainage volume of $>1500 \mathrm{ml}$ over 2 weeks should be surgically ligated earlier [23].

In 1999 Cerfolio et al. pointed out that thoracostomy drainage of $>1000 \mathrm{ml} /$ day for the first 7 days and the 
development of a chylothorax after an esophageal operation were significant factors indicating an earlier need for reoperation [5]. Most recently (2002) Cope and Kaiser stated that in patients with unremitting or recurrent chyle output of $<500 \mathrm{ml} /$ day, successful management is possible with chest drainage and a low-fat diet, administered orally or parenterally, with the favorable result being that the chyle fistula seals off after 2-3 weeks, with or without pleurodesis [24]. According to these data we chose a maximum of 3 weeks as the upper threshold for a conservative therapeutic approach to determine its effects.

Conservative treatment in cases of chylothorax means closed chest drainage or intermittent thoracocentesis supported by medium-chain triglycerides given orally or even total parenteral nutrition. Persistent high output may be treated with i.v. somatostatin-14 or etilefrin, pleurodesis, or percutaneous embolization [3-6]. In cases of superficial lymphocele or lymphatic fistula, external compression may be included. Lüchtenberg et al. reported a case in which the use of lipiodol in dacryocystography with complicating perforation led to granulomatous reaction and consecutive phlegmone, showing the potential of lipiodol to induce inflammatory, granulomatous processes when extravasating [2]. In cases which may not lead to occlusion of the perforation with conservative treatment alone, initial LAG is further helpful by showing the surgeon the precise site and height at which the lymphatic vessel ligation needs to be performed, thereby simplifying preoperative planning and reducing unexpected complications that may occur during intraoperative exploration [25]. In addition, there are rare cases described in which LAG in patients with known chylothorax revealed spontaneous occlusion of the leaking thoracic duct, making further surgical intervention unnecessary [25]. Our study proves that, at $75 \%$, the sensitivity of pedal lipiodol lymphangiograpy to directly determine the sites of femoral, thoracic, and abdominal contrast leakage, fistula, or lymphocele, respectively, is very good. In the remaining $25 \%$ of cases, it was at least able to identify lymphatic vessel injury. Having shown that $73.3 \%$ of the patients who were treated conservatively and not reoperated earlier than 3 weeks after LAG did not need any reoperation at all, we confirm that LAG is a highly effective and powerful tool even in a therapeutic approach. As shown in rare cases, it may also reveal relevant pathologies (e.g., foreign bodies) adjacent to the lymphatic system.

Apart for these advantages, we must mention that there are strict contraindications for the procedure, such as pulmonary insufficiency or right-to-left cardiac shunt, the first being in risk of exacerbation by pulmonary embolism through oil droplets, the latter creating the risk of cerebral embolism [26]. LAG also should not be performed in cases of lymphedema. Allergic reactions to patent blue dye have been described, which may be treated with i.v. H1/H2- anthistamines (clemastin, ranitidin) and i.v. steroids (methyl-prednisolone) [27].

\section{Conclusion}

Our study clearly demonstrates that even in the era of modern cross-sectional imaging, classic LAG is a powerful and highly reliable tool to visualize and even assist occlusion of the postoperatively damaged lymphatic vessel. Thereby, the need for reoperation and exposure to perioperative mortality may be avoided. Concerning the small numbers in our series, especially in the subgroup analysis, of course, these results are preliminary and need further confirmation.

\section{References}

1. Guermazi A, Brice P, Hennequin C, et al. (2003) Lymphography: an old technique retains its usefulness. Radiographics 23(6):1541-1558, discussion 1559-1560

2. Luchtenberg M, Mikowski A, Schalnus R, et al. (2000) Erythematous eyelid swelling after dacryocystography. Perforation of the efferent lacrimal ducts in dacryocystography with contrast medium extravasation. Ophthalmologe 97(12):894-895

3. Browse NL, Allen DR, Wilson NM (1997) Management of chylothorax. Br J Surg 84(12):1711-1716

4. Cope C, Salem R, Kaiser LR (1999) Management of chylothorax by percutaneous catheterization and embolization of the thoracic duct:prospective trial. J Vasc Interv Radiol 10(9):1248-1254

5. Cerfolio RJ, Allen MS, Deschamps C, et al. (1996) Postoperative chylothorax. J Thorac Cardiovasc Surg 112(5):1361-1365, discussion 1365-1366

6. Hoffer EK, Bloch RD, Mulligan MS, et al. (2001) Treatment of chylothorax: percutaneous catheterization and embolization of the thoracic duct. AJR 176(4):1040-1042

7. Sachs PB, Zelch MG, Rice TW, et al. (1991) Diagnosis and localization of laceration of the thoracic duct: usefulness of LAG and CT. AJR Am J Roentgenol, 157(4):703-5

8. Halloul Z, Meyer F, Burger T, et al. (1995) Chylous ascites, a rare complication of aortic surgery. Vasa 24(4):377-381

9. Allen W, Parrott TS, Saripkin L, et al. (1986) Chylous ascites following retroperitoneal lymphadenectomy for granulosa cell tumor of the testis. J Urol 135(4):797-798

10. Jansen TT, Debruyne FM, Delaere KP, et al. (1984) Chylous ascites after retroperitoneal lymph node dissection. Urology 23(6):565-567

11. Campieri C, Raimondi C, Dalmastri V, et al. (1996) Posttraumatic chyluria due to lymphorenal fistula regressed after somatostatin therapy. Nephron 72(4):705-707

12. Peh WC, Ooi GC, Ngan H (1996) Case quiz. Lymphographic demonstration of chylothorax. Australas Radiol 40(4):463-464

13. Shermak MA, Yee K, Wong L, et al. (2005) Surgical management of groin lymphatic complications after arterial bypass surgery. Plast Reconstr Surg 115(7):1954-1962

14. Karcaaltincaba M, Akhan O (2005) Radiologic imaging and percutaneous treatment of pelvic lymphocele. Eur J Radiol 55(3):340-354

15. Canovas B, Morlan MA, Familiar C, et al. (2005) Resolution of a neck chylous fistula with oral diet treatment. Nutr Hosp 20(6):429-432 
16. Heilmann RD, Collins VP (1963) Identification of laceration of the thoracic duct by LAG. Radiology 81:470-472

17. Samaniego V, Moguel R, Meaney E, et al. (1993) Massive chylopericardium due to congenital fistulas. Am Heart $\mathbf{J}$ 126(4):1005-1006

18. Jarman PR, Whyte K, Sabroe I, et al. (1995) Sarcoidosis presenting with chylothorax. Thorax 50(12):1324-1325

19. Dugue L, Sauvanet A, Farges O, et al. (1998) Output of chyle as an indicator of treatment for chylothorax complicating oesophagectomy. Br J Surg 85(8):1147-1149

20. Alexiou C, Watson M, Beggs D, et al. (1998) Chylothorax following oesophagogastrectomy for malignant disease. Eur J Cardiothorac Surg 14(5):460-466

21. Wurnig PN, Hollaus PH, Ohtsuka T, et al. (2000) Thoracoscopic direct clipping of the thoracic duct for chylopericardium and chylothorax. Ann Thorac Surg 70:1662-1665
22. Maloney JV, Spencer FC (1956) The nonoperative treatment of traumatic chylothorax. Surgery 40(1):121-128

23. Selle JG, Snyder WH (1971) Chylothorax: indication for surgery. Ann Surg 177:245-249

24. Cope C, Kaiser LR (2002) Management of unremitting chylothorax by percutaneous embolization and blockage of retroperitoneal lymphatic vessel leakage. J Vasc Int Radiol 13:1139-1148

25. Ngan H, Fok M, Wong J (1988) The role of lymphography in chylothorax following thoracic surgery. $\mathrm{Br} \mathrm{J}$ Radiol 61(731):1032-1036

26. Kusumoto S, Imamura A, Watanabe K (1991) Case report:the incidental lipid embolization to the brain and kidney after lymphography in a patient with malignant lymphoma: CT findings. Clin Radiol 44(4):279-280

27. Mortazavi SH, Burrows BD (1971) Allergic reaction to patient blue dye in LAG. Clin Radiol 22(3):389-390 